\title{
Review
}

\section{The Role of Transient Receptor Potential Channels in Metabolic Syndrome}

\author{
Daoyan LIU'), Zhiming ZHU'1), and Martin TEPEL ${ }^{2)}$
}

\begin{abstract}
Metabolic syndrome is correlated with increased cardiovascular risk and characterized by several factors, including visceral obesity, hypertension, insulin resistance, and dyslipidemia. Several members of a large family of nonselective cation entry channels, e.g., transient receptor potential (TRP) canonical (TRPC), vanilloid (TRPV), and melastatin (TRPM) channels, have been associated with the development of cardiovascular diseases. Thus, disruption of TRP channel expression or function may account for the observed increased cardiovascular risk in metabolic syndrome patients. TRPV1 regulates adipogenesis and inflammation in adipose tissues, whereas TRPC3, TRPC5, TRPC6, TRPV1, and TRPM7 are involved in vasoconstriction and regulation of blood pressure. Other members of the TRP family are involved in regulation of insulin secretion, lipid composition, and atherosclerosis. Although there is no evidence that a single TRP channelopathy may be the cause of all metabolic syndrome characteristics, further studies will help to clarify the role of specific TRP channels involved in the metabolic syndrome. (Hypertens Res 2008; 31: 1989-1995)
\end{abstract}

Key Words: metabolic syndrome, transient receptor potential channel, hypertension, cardiometabolic risk

\section{Metabolic Syndrome}

Metabolic syndrome is associated with several major risk factors, including visceral obesity, hypertension, insulin resistance and dyslipidemia (1). Although the National Cholesterol Education Program's Adult Treatment Panel III (ATP-III) (2-4), the World Health Organization (5), and the International Diabetes Federation (6) have proposed different diagnostic criteria, all the definitions include measures of anthropometric body composition, blood pressure, glucose, and lipids. According to the modified NCEP-ATP III report (2-4), metabolic syndrome is defined as having three or more of the following conditions: abdominal obesity with waist circumference $\geq 90 \mathrm{~cm}$ in men and $\geq 80 \mathrm{~cm}$ in women; high blood pressure including systolic blood pressure $\geq 130 \mathrm{mmHg}$ or diastolic blood pressure $\geq 85 \mathrm{mmHg}$; high fasting blood glucose $\geq 110 \mathrm{mg} / \mathrm{dL}(6.1 \mathrm{mmol} / \mathrm{L})$; hypertriglyceridemia $\geq 150 \mathrm{mg} / \mathrm{dL}$ (1.7 mmol/L); or high-density lipoprotein cholesterol $<40 \mathrm{mg} / \mathrm{dL}(1.0 \mathrm{mmol} / \mathrm{L})$ in $\mathrm{men}$ or $<50 \mathrm{mg} / \mathrm{dL}(1.29$ $\mathrm{mmol} / \mathrm{L})$ in women.

Metabolic syndrome is indicative of increased cardiovascular risk. In the Multiple Risk Factor Intervention Trial (MRFIT), men with metabolic syndrome had significantly higher risk for 18-year total mortality (hazard ratio, 1.21), cardiovascular disease mortality (hazard ratio, 1.49), and coronary heart disease mortality (hazard ratio, 1.51) relative to those without metabolic syndrome (7). The National Health and Nutrition Examination Survey III (NHANES III) showed an association between metabolic syndrome and a history of myocardial infarction or stroke $(8)$. In a prospective cohort study of 6,255 subjects, the hazard ratio for cardiovascular

From the ${ }^{1)}$ Center for Hypertension and Metabolic Diseases, Department of Hypertension and Endocrinology, Daping Hospital, Third Military Medical University, Chongqing, China; and ${ }^{2}$ Department of Nephrology, Medical Clinic, Charité Campus Benjamin Franklin, Berlin, Germany.

This work was funded by grants from the Natural Science Foundation of China (No. 30871058 to D.L.) and from the National Basic Research Program of China (No. 2006CB503804 and 2006CB503905 to Z.Z.). M.T. received a grant from Deutsche Forschungsgemeinschaft (DFG TE 150/21-1).

Address for Reprints: Zhiming Zhu, M.D., Ph.D., Center for Hypertension and Metabolic Diseases, Department of Hypertension and Endocrinology, Daping Hospital, Third Military Medical University, Chongqing, China. E-mail: zhuzming@mail.dph-fsi.com; Daoyan Liu, Ph.D., Center for Hypertension and Metabolic Diseases, Daping Hospital, Third Military Medical University, Chongqing Institute of Hypertension, Chongqing, China. E-mail: ldy_liudaoyan@yahoo.com

Received July 13, 2008; Accepted in revised form September 3, 2008. 


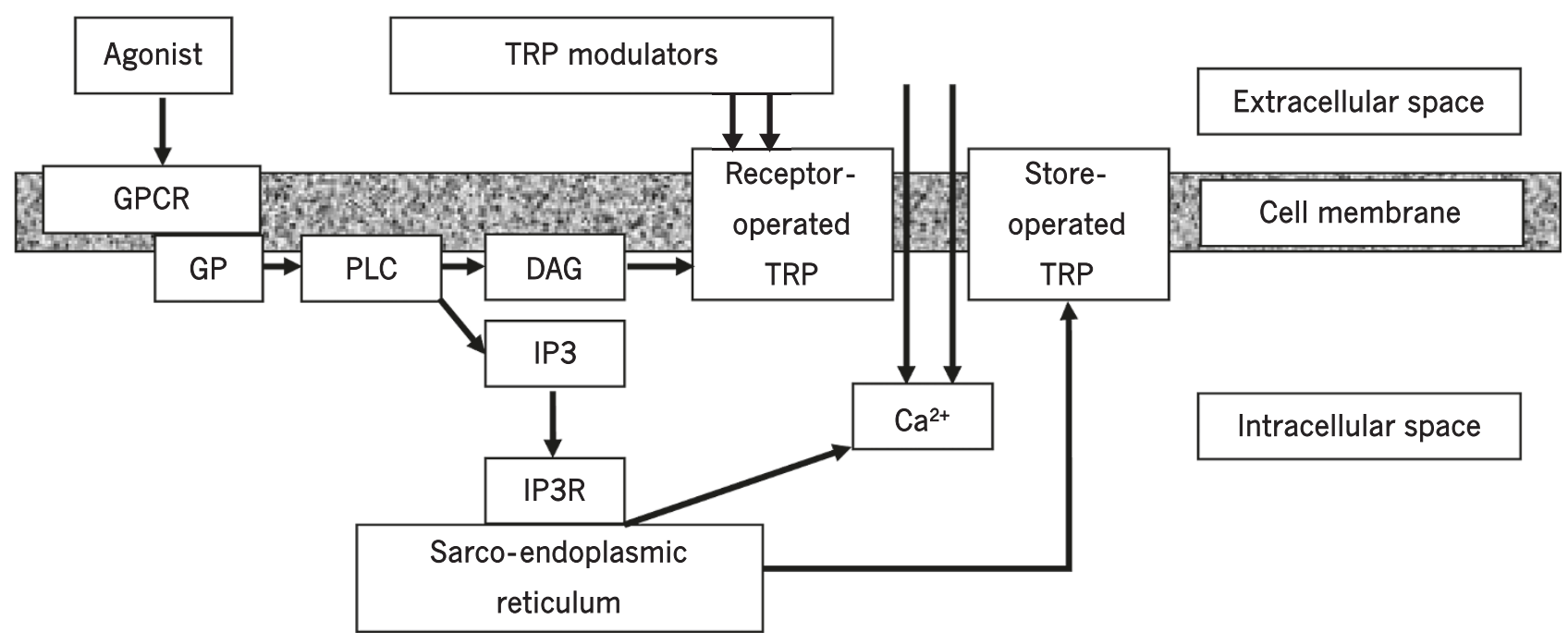

Fig. 1. Involvement of transient receptor potential (TRP) channels in cellular calcium homeostasis. The activation of G-protein coupled receptors (GPCR) by several agonists, e.g. angiotensin II, and subsequent release of G proteins (GP) stimulates phospholipase C $\beta 1$ (PLC) to produce diacylglycerol (DAG) and inositoltrisphosphate (IP3). DAG may activate receptor-operated TRP channels directly, whereas other store-operated TRP channels are activated after IP3-receptor (IP3R) induced calcium release from intracellular stores, i.e. sarco-endoplasmic reticulum. Endogenous and exogenous modulators of TRP channels may regulate TRP channel activity.

disease mortality was $2.02(95 \%$ confidence interval [CI], 1.42 to 2.89 ) for those with metabolic syndrome compared to those without, after adjusting for age, gender and risk facter (9). Additionally, metabolic syndrome criteria have recently been used to identify diabetic patients at greater risk for macrovascular complications. However, because a significant overlap was seen in 10-year estimated cardiovascular risk in diabetic patients with or without metabolic syndrome, the authors of this study concluded that a diagnosis of metabolic syndrome holds limited clinical value for assessing risk in diabetic patients (10).

A large, new family of non-selective cation entry channels called the transient receptor potential (TRP) channels has been associated with many diverse of biological functions. TRP channel subunits contain six hydrophobic stretches and a pore loop motif intercalated between the fifth and sixth putative transmembrane segments, strongly reminiscent of single of the four intramolecularly linked transmembrane domains in voltage-gated $\mathrm{Ca}^{2+}$ and $\mathrm{Na}^{+}$channels. Therefore, TRP channels generally belong to the large superfamily of hexahelical cationic channels. The mammalian TRP channel family core consists of, subfamilies of classical TRP channels (TRPC1-TRPC7), vanilloid receptor-related TRP channels (TRPV1-TRPV6), melastatin-related TRP channels (TRPM1-TRPM8) and polycystin-related TRP channels (TRPP1-TRPP2) (11). TRP channels are involved in visual, auditory, taste, and pain signal transduction pathways, regulation of blood circulation, gut motility, mineral absorption, body fluid balance, airway and bladder hypersensitivities, cell survival and growth $(12,13)$. TRP channels have six transmembrane segments with a putative pore loop between the fifth and sixth transmembrane domains. This structure resembles voltage-dependent cation channels but lacks the positively charged amino acid residues characteristic for voltagesensing (13). Several subtypes of canonical TRPC channels are activated by $\mathrm{G}$ protein-coupled receptors and receptor tyrosine kinases that are linked to phosphoinositide hydrolysis via phospholipase $\mathrm{C}$ activation, whereas other TRPC channel subtypes are activated only after depletion of intracellular calcium stores. In addition, vanilloid-related TRPV channels are also affected by pungent agents, including capsaicin or capsazepine, lipids, heat, shear stress or hypoosmolarity. Furthermore, melastatin-related TRPM channels are activated by oxidative stress, cold exposure and cooling agents such as menthol $(12,13)$.

TRP channels have been associated with several diseases and implicated specifically in the development of several cardiovascular diseases $(12,13)$. TRP channels are broadly distributed in several cell types, including adipocytes, vascular smooth muscle cells and endothelial cells, which explains their wide spread function. Thus changes in expression or disturbance of TRP channel function likely result in the development of metabolic syndrome. Several channels including TRPC3, TRPC6, TRPC7, and TRPV1 are activated primarily by diacylglycerol, whereas other channels including TRPC4, TRPC5, TRPC6, and TRPC7 are activated only after depletion of intracellular sarcoendoplasmic stores. Several agonists implicated in the pathophysiology of the metabolic syndrome 
may directly affect TRP channels. For example, angiotensin II activates the angiotensin receptor subtype 1 (AT1) attached

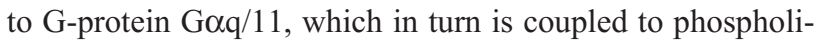
pase $C \beta 1$. Activation of phospholipase $C \beta 1$ enhances the cleavage of phosphoinositides into inositol trisphosphate and diacylglycerol. Both depletion of inositol trisphosphate stores and elevation of diacylglycerol activate TRP channels, as depicted in Fig. 1. Changes in TRP channel expression or heteromer composition may thus cause changes in transplasma membrane calcium influx, intracellular calcium homeostasis and cellular functions involved in metabolic syndrome.

\section{Obesity}

Obesity is a hallmark of metabolic syndrome. Several experiments indicate that TRPV1 channel interactions can affect preadipocytes, obesity-induced chronic inflammatory responses, fat distribution via afferent autonomic nerves and appetite regulation. Several endogenous agonists ( $N$-arachidonyl dopamine and anandamide) as well as exogenous agonists (capsaicin and resiniferatoxin) are currently available and may modulate TRPV1 channel activity.

Zhang et al. (14) have previously demonstrated the presence of TRPV1 channels in preadipocytes and visceral adipose tissues both mice and humans. The TRPV1-agonist capsaicin induced calcium influx in a dose-dependent manner and prevented adipogenesis in stimulated preadipocytes. Reduced TRPV1 expression was observed in visceral adipose tissues from obese mice and human male subjects when compared with their lean counterparts. Furthermore, administration of the TRPV1-agonist capsaicin prevented obesity in male wild-type mice but not in TRPV1 knockout mice assigned a high-fat diet (14). Capsaicin activation of TRPV1 in 3T3-L1 preadipocytes reduced adipogenesis by decreasing the activities of peroxisome proliferator-activated receptor $\gamma$ (PPAR $\gamma)$, CCAAT/enhancer-binding protein (C/EBP $\alpha)$, leptin, intracellular triglycerides, and glycerol-3-phosphate dehydrogenase activity. Furthermore activation of TRPV1 in 3T3-L1 preadipocytes increased apoptosis by activation of caspase-3, pro-apoptotic Bax and Bak, cleavage of poly(ADP-ribose) polymerase (PARP), and downregulation of anti-apoptotic Bcl-2 (15). Capsaicin also activated the serine/threonine kinase, AMP-activated kinase (AMPK), in 3T3-L1 preadipocytes, causing reduced adipogenesis and increased apoptosis (16). Activation of TRPV1 by capsaicin led to PPAR $\gamma$ activation, nuclear factor- $\kappa$ B (NF- $\kappa B)$ inactivation, suppression of obesity-associated macrophage migration, and the release proinflammatory mediators by macrophage activation. Uncoupling proteins also play a very important role in thermogenesis and fatty acid oxidation in adipose tissues. Activation of TRPV1 by capsaicin and capsiate, a nonpungent capsaicin analog, upregulated expression of uncoupling protein (UCP) 1 and UCP2 expression in brown and white adipose tissues in rodents (17). Obesity-induced chronic inflammatory responses play crucial roles in the development of type II diabetes mellitus and atherosclerosis (18). Treating 1.5-month-old rats with the TRPV1 with the TRPV1 agonist capsaicin destroyed capsaicin-sensitive afferent autonomic nerves and reduced circulating calcitonin gene-related peptide (CGRP). CGRP is primarily secreted by TRPV1-expressing nerves and antagonizes insulin release. Persistently high circulating CGRP levels cause insulin resistance and resultant obesity in the long term. Additionally, white adipose tissue was significantly reduced in capsaicintreated rats 1 year after treatment compared to control rats (19). Endogenous TRPV1 ligands, such as $N$-oleoylethanolamide, reduced food intake in wild-type, but not in TRPV1 knockout mice, indicating that activation of vagal sensory afferent neurones may regulate appetite and caloric intake (20). Bratz et al. (21) recently compared TRPV1 channel activation in coronary arteries from lean and obese male Ossabaw miniature swines and showed that TRPV1 channels are functionally expressed in the coronary circulation. TRPV1 channels were also found to mediate endothelium-dependent vasodilatation through a mechanism involving nitric oxide and potassium channels. Furthermore, impaired capsaicininduced vasodilatation in metabolic syndrome was associated with decreased expression of TRPV1 and cation influx (21). These results further support a link between impaired TRPV1 channels and cardiovascular dysfunction in the pathogenesis of metabolic syndrome.

\section{Diabetes}

Experimental evidence indicates that several TRP channels play an important role in the onset of diabetes mellitus. Razavi et al. reported that activation of TRPV1-positive pancreatic sensory neurons controls islet inflammation and insulin resistance. After elimination of TRPV1-positive pancreatic sensory neurons in diabetes-prone mice, the development of insulitis and diabetes could be prevented, indicating that TRPV1 activation is associated with the pathogenesis of type 1 diabetes mellitus (22). Additionally, TRPC4-like channels provide a pathway for depolarization and calcium influx into insulin-secreting cells (23). TRPM2 channels are also directly involved in insulin secretion from pancreatic islets. TRPM2-induced calcium entry into pancreatic islets, was increased in the presence of cyclic ADP-ribose, thereby regulating insulin secretion (24). Insulin-resistant cardiomyocytes obtained from ob/ob mice displayed reduced TRPC3 expression and impaired diacylglycerol-induced nonselective cation currents (25). Furthermore, high glucose concentrations reduced TRPC6 expression in mesangial cells (26). However, a recent study demonstrated that hyperglycemia significantly increased TRPC6 protein expression in platelets, whereas expressions of TRPC1, TRPC3, TRPC4 and TRPC5 remained unchanged (27). This latter finding is in agreement with previous results indicating that patients with diabetes mellitus are prone to cardiovascular complications, including microangiopathy, retinopathy, and nephropathy, which can 


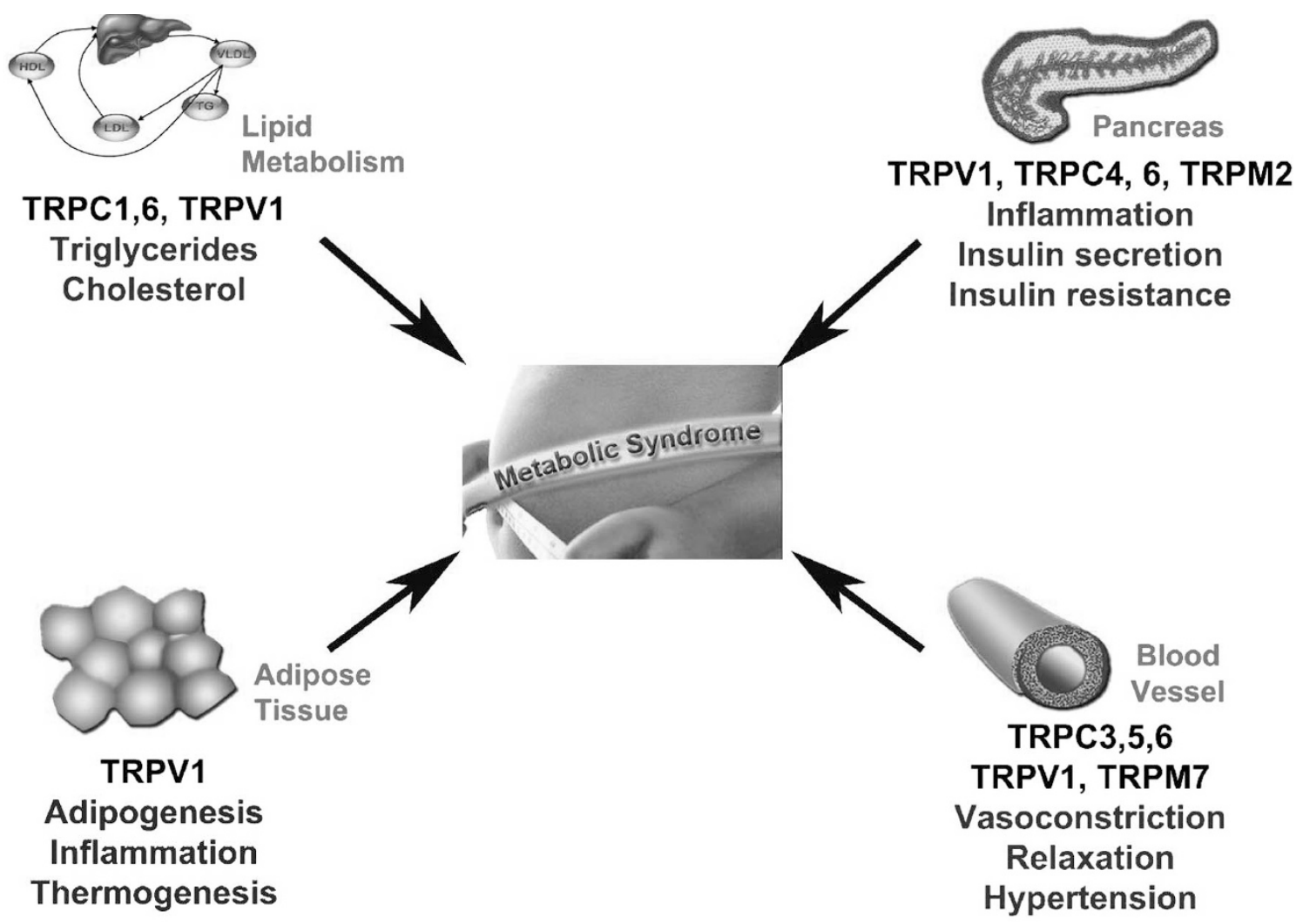

Fig. 2. Involvement of transient receptor potential (TRP) canonical (TRPC), vanilloid (TRPV) and melastatin (TRPM) channels in target organs of the metabolic syndrome. TRP channels activation affect multiple organs involved in the metabolic syndrome through modulation of the intracellular calcium homeostasis in tissue- and cell-specific fashion. TRPV1 regulates adipogenesis and inflammation in adipose tissue. In pancreatic cells TRPV1, TRPC4, TRPC6 and TRPM2 participate in inflammation and insulin secretion. In the vasculature TRPC3, TRPC5, TRPC6, TRPV1, and TRPM7 are involved in the vasoconstriction, relaxation and regulation of blood pressure. TRPC1, TRPC6, and TRPV1 also influence serum lipids and membrane cholesterol metabolism.

be attributed in part to increased platelet activity in diabetics. Taken together, TRP channels are involved either in islet inflammation and impaired insulin secretion or in the pathogenesis of diabetic complications.

\section{Hypertension}

Data from both animal models and patients with essential hypertension indicate that TRP channels are directly involved in the pathogenesis of primary hypertension. Liu et al. demonstrated increased TRPC 3 expression in peripheral monocytes from spontaneously hypertensive rats (SHR) compared to normotensive Wistar-Kyoto (WKY) rats (28). After TRPC3 siRNA knockdown, thapsigargin-induced store-operated calcium influx and 1-oleoyl-2-acetyl-sn-glycerolinduced calcium influx were both significantly reduced in SHR cells compared to those in WKY cells (29). In addition, TRPC3 expression in TRPC6 knockout mice was found to be greatly enhanced although TRPC3 upregulation was not func- tionally interchangeable with TRPC6 (30). TRPC6 knockout mice displayed increased TRPC 3 channel expression. Furthermore, TRPC6 knockout mice show elevated blood pressure and increased arterial vasoconstriction compared to wild-type counterparts. Based on these data, TRPC 3 channels were suggested to have constitutive activities, while TRPC6 remains almost quiescent in the absence of any agonistinduced receptor stimulation (31). Thus, increased TRPC3 expression relative to that of TRPC6 may predispose mice to vasoconstriction and hypertension.

TRPC6 channels may also directly contribute to salt-sensitive hypertension. In rat aortic smooth muscle cells, agonistmediated activation of TRPC 6 channels caused an increase of localized cytosolic sodium transients at the cell periphery in subsarcolemmal, cytoplasmic microdomains, as measured by the sodium indicator CoroNa-Green. This increase of localized cytosolic sodium then enhances calcium influx mediated by the sodium/calcium exchanger (32). Bae et al. reported a significant increase in TRPC6 expression and receptor-stimu- 
lated currents in mesenteric arterial smooth muscle cells of deoxycorticosterone acetate-salt hypertensive rats when compared to control rats (33). Reduced TRPV1 expression was reported in Dahl salt-sensitive rats that develop hypertension after salt feeding, rendering these animals sensitive to salt load in blood pressure regulation (34). TRPM7 was downregulated in mesenteric arterial smooth muscle cells from SHR. Because TRPM7 preferentially mediates agonist-induced transplasma membrane magnesium influx, reduced TRPM7 expression is associated with reduced cytosolic magnesium concentrations, which may facilitate vasoconstriction (35).

Thilo et al. demonstrated an 8-fold increase of TRPC3 transcripts in monocytes from human patients with essential hypertension compared to normotensive control subjects. Furthermore, a correlation between TRPC3 transcripts with systolic blood pressure was observed (36). Increased TRPC3 and TRPC5 protein expression was also observed in patients with essential hypertension when compared to normotensive control subjects matched in age and gender (37).

Increased TRPC expression in patients with essential hypertension was associated with increased calcium influx. Store-operated calcium influx was reduced by the 2 -aminoethoxy-diphenylborane inhibitor when TRPC3 and TRPC5 (but not TRPC6) were knocked down with siRNA. Furthermore, receptor-operated 1-oleoyl-2-acetyl-sn-glycerol-induced calcium and barium influxes were significantly elevated in essential hypertension patients, while 1-oleoyl-2-acetyl-snglycerol-induced cation influx was reduced when TRPC3 and TRPC5 were specifically knocked down (38). Erythropoietin, a glycoprotein required for proliferation and differentiation of erythroid cells, was shown to modulate TRPC3 activation through a phospholipase $\mathrm{C} \gamma$-mediated process requiring interaction of phospholipase $\mathrm{C} \gamma$ and inositol 1,4,5trisphosphate receptor with TRPC3 (39). Patients with metabolic syndrome and hypertension showed a higher red blood cell count and increased erythropoietin, hemoglobin, and hematocrit levels (40). Taking into account the cardiovascular effects of erythropoietin, an association between erythropoietin and TRPC3 may also contribute to the development of hypertension in patients with metabolic syndrome (41).

\section{Lipids}

Several studies have shown that TRP channels affect lipid metabolism (42-46). Activation of TRPV1 channels by capsaicin resulted in reduced cellular triglyceride levels in 3T3L1 preadipocytes during adipogenesis in vitro (14). Activation of TRPV1 channels by capsaicin also reduced dietary high-fat-induced hypertriglyceridemia in rats on a diet containing 30\% fat for 8 weeks (42). Rats fed a high-fat diet displayed higher lipoprotein lipase activity in adipose tissues after capsaicin administration (43).

Membrane lipid content can influence cellular TRP channel distribution, localization, and function. Bergdahl et al. reported that reduction of membrane cholesterol using the cholesterol acceptor methyl- $\beta$-cyclodextrin in intact rat arteries reduced the expression of TRPC1 transcript levels, colocalization of TRPC1 with caveolin-1 by immunofluorescence, and contractile responsiveness to endothelin-1 (44). Similarly, cholesterol depletion using methyl- $\beta$-cyclodextrin reduced TRPV1 protein levels in membrane fractions and TRPV1-mediated capsaicin- and proton-activated currents in adult rat dorsal root ganglion neuros (45). Furthermore, in cultured human corporal smooth muscle cells, lysophosphatidylcholine has been shown to activate TRPC6 channels and calcium influx in cultured human corporal smooth muscle cells (46). Incorporation of 7-ketocholesterol, a component of oxidized LDL, into lipid raft domains of monocytic cell plasma membranes caused TRPC1 translocation to rafts and increased transplasma membrane calcium influx (47).

\section{Atherosclerosis}

Development of atherosclerosis is common in metabolic syndrome. Progression of atherosclerosis is influenced by several lipid factors. TRPC3 and TRPC5 channels have been shown to be involved in sensing of lipids, reactive oxygen species and reactive nitrogen species (48). Kumar et al. reported that upregulation of TRPC1 is a general feature of vascular injury and neointimal hyperplasia and that TRPC1 channel inhibitors may protect against occlusive vascular disease (49). A recent study showed that activation of angiotensin II and nuclear factor NF- $\mathrm{KB}$ induced human coronary artery smooth muscle cell hypertrophy through enhanced TRPC1 channel expression (50). Current studies suggested that dysregulations of TRPC4 and TRPC1 channels is associated with damaged vascular endothelial barriers, while abnormalities in TRPP1 and TRPP2 caused impaired endothelial nitric oxide synthase production. Reduced expression of TRPC4 and TRPV1 inhibited vascular relaxation, with TRPV4 dysfunction reducing flow-induced vascular responses. Finally, both TRPC3 and TRPC4 are associated with oxidative stressinduced endothelial disturbances (51). These examples underscore the relevance of TRP channels in the pathogenesis of atherosclerosis.

In summary, disruption in expression or function of TRP channels has been associated with metabolic syndrome (Fig. $2)$. However, there is no current data suggasting that one single TRP channelopathy may underlie all metabolic syndrome characteristics. Undoubtedly, the design and development of TRP agonists and antagonists $(52,53)$ will help to further clarify the role of specific TRP channels in metabolic syndrome.

\section{References}

1. Eckel RH, Grundy SM, Zimmet PZ: The metabolic syndrome. Lancet 2005; 365: 1415-1428.

2. Expert Panel on Detection Evaluation and Treatment of High Blood Cholesterol in Adults: Executive Summary of 
the Third Report of the National Cholesterol Education Program (NCEP) Expert Panel on Detection, Evaluation, and Treatment of High Blood Cholesterol in Adults (Adult Treatment Panel III). JAMA 2001; 285: 2486-2497.

3. Chiba Y, Saitoh S, Takagi S, et al: Relationship between visceral fat and cardiovascular disease risk factors: the Tanno and Sobetsu Study. Hypertens Res 2007; 30: 229236.

4. Scott M, Grundy SM, Cleeman JI, et al: Diagnosis and Management of the Metabolic Syndrome: an American Heart Association/National Heart, Lung, and Blood Institute Scientific Statement. Circulation 2005; 112: 27352752.

5. World Health Organization: Definition, Diagnosis and Classification of Diabetes Mellitus and Its Complications: Report of a WHO Consultation, Part 1: Diagnosis and Classification of Diabetes Mellitus. Geneva, World Health Organization, 1999.

6. Alberti KG, Zimmet P, Shaw J: The metabolic syndrome: a new worldwide definition. Lancet 2005; 366: 1059-1062.

7. Eberly LE, Prineas R, Cohen JD, et al, Multiple Risk Factor Intervention Trial Research Group: Metabolic syndrome: risk factor distribution and 18-year mortality in the multiple risk factor intervention trial. Diabetes Care 2006; 29: $123-$ 130.

8. Ninomiya JK, L'Italien G, Criqui MH, Whyte JL, Gamst A, Chen RS: Association of the metabolic syndrome with history of myocardial infarction and stroke in the Third National Health and Nutrition Examination Survey. Circulation 2004; 109: 42-46.

9. Malik S, Wong ND, Franklin SS, et al: Impact of the metabolic syndrome on mortality from coronary heart disease, cardiovascular disease, and all causes in United States adults. Circulation 2004; 110: 1245-1250.

10. Cull CA, Jensen CC, Retnakaran R, Holman RR: Impact of the metabolic syndrome on macrovascular and microvascular outcomes in type 2 diabetes mellitus: United Kingdom Prospective Diabetes Study 78. Circulation 2007; 116: 2119-2126.

11. Schaefer M: Homo- and heteromeric assembly of TRP channel subunits. Pflügers Arch 2005; 451: 35-42.

12. Nilius B, Owsianik G, Voets T, Peters JA: Transient receptor potential cation channels in disease. Physiol Rev 2007; 87: $165-217$.

13. Inoue R, Jensen LJ, Shi J, et al: Transient receptor potential channels in cardiovascular function and disease. Circ Res 2006; 99: 119-131.

14. Zhang LL, Liu DY, Ma LQ, et al: Activation of transient receptor potential vanilloid type-1 channel prevents adipogenesis and obesity. Circ Res 2007; 100: 1063-1070.

15. Hsu CL, Yen GC: Effects of capsaicin on induction of apoptosis and inhibition of adipogenesis in 3T3-L1 cells. $J$ Agric Food Chem 2007; 55: 1730-1736.

16. Hwang JT, Park IJ, Shin JI, et al: Genistein, EGCG, and capsaicin inhibit adipocyte differentiation process via activating AMP-activated protein kinase. Biochem Biophys Res Commun 2005; 338: 694-699.

17. Kang JH, Kim CS, Han IS, Kawada T, Yu R: Capsaicin, a spicy component of hot peppers, modulates adipokine gene expression and protein release from obese-mouse adipose tissues and isolated adipocytes, and suppresses the inflammatory responses of adipose tissue macrophages. FEBS Lett 2007; 581: 4389-4396.

18. Masuda Y, Haramizu S, Oki K, et al: Upregulation of uncoupling proteins by oral administration of capsiate, a nonpungent capsaicin analog. J Appl Physiol 2003; 95: 2408-2415.

19. Melnyk A, Himms-Hagen J: Resistance to aging-associated obesity in capsaicin-desensitized rats one year after treatment. Obes Res 1995; 3: 337-344.

20. Wang X, Miyares RL, Ahern GP: Oleoylethanolamide excites vagal sensory neurones, induces visceral pain and reduces short-term food intake in mice via capsaicin receptor TRPV1. J Physiol 2005; 564: 541-547.

21. Bratz IN, Dick GM, Tune JD, et al: Impaired capsaicininduced relaxation of coronary arteries in a porcine model of the metabolic syndrome. Am J Physiol Heart Circ Physiol 2008; 294: H2489-H2496.

22. Razavi R, Chan Y, Afifiyan FN, et al: TRPV1+ sensory neurons control beta cell stress and islet inflammation in autoimmune diabetes. Cell 2006; 127: 1123-1135.

23. Qian F, Huang P, Ma L, Kuznetsov A, Tamarina N, Philipson LH: TRP genes: candidates for nonselective cation channels and store-operated channels in insulin-secreting cells. Diabetes 2002; 51 (Suppl 1): S183-S189.

24. Togashi K, Hara Y, Tominaga T, et al: TRPM2 activation by cyclic ADP-ribose at body temperature is involved in insulin secretion. EMBO J 2006; 25: 1804-1815.

25. Fauconnier J, Lanner JT, Sultan A, et al: Insulin potentiates TRPC3-mediated cation currents in normal but not in insulin-resistant mouse cardiomyocytes. Cardiovasc Res 2007; 73: $376-385$.

26. Graham S, Ding M, Sours-Brothers S, Yorio T, Ma JX, Ma R: Downregulation of TRPC6 protein expression by high glucose, a possible mechanism for the impaired $\mathrm{Ca}^{2+}$ signaling in glomerular mesangial cells in diabetes. Am J Physiol Renal Physiol 2007; 293: F1381-F1390.

27. Liu D, Maier A, Scholze A, et al: High glucose enhances transient receptor potential channel canonical type 6 (TRPC6)-dependent calcium influx in human platelets via phosphatidylinositol 3-kinase-dependent pathway. Arterioscler Thromb Vasc Biol 2008; 28: 746-751.

28. Liu DY, Scholze A, Zhu ZM, et al: Increased transient receptor potential channel TRPC3 expression in spontaneously hypertensive rats. Am J Hypertens 2005; 18: $1503-$ 1507.

29. Liu DY, Scholze A, Kreutz R, et al: Monocytes from spontaneously hypertensive rats show increased store-operated and second messenger-operated calcium influx mediated by transient receptor potential canonical type 3 channels. Am J Hypertens 2007; 20: 1111-1118.

30. Dietrich A, Mederos Y, Schnitzler M, et al: Increased vascular smooth muscle contractility in $\mathrm{TRPC}^{-/-}$mice. $\mathrm{Mol}$ Cell Biol 2005; 25: 6980-6989.

31. Trebak M, Vazquez G, Bird GS, Putney JW Jr: The TRPC3/ 6/7 subfamily of cation channels. Cell Calcium 2003; 33: 451-461.

32. Poburko D, Liao CH, Lemos VS, et al: Transient receptor potential channel 6-mediated, localized cytosolic $\left[\mathrm{Na}^{+}\right]$ transients drive $\mathrm{Na}^{+} / \mathrm{Ca}^{2+}$ exchanger-mediated $\mathrm{Ca}^{2+}$ entry in 
purinergically stimulated aorta smooth muscle cells. Circ Res 2007; 101: 1030-1038.

33. Bae YM, Kim A, Lee YJ, et al: Enhancement of receptoroperated cation current and TRPC6 expression in arterial smooth muscle cells of deoxycorticosterone acetate-salt hypertensive rats. J Hypertens 2007; 25: 809-817.

34. Wang Y, Wang DH: A novel mechanism contributing to development of Dahl salt-sensitive hypertension: role of the transient receptor potential vanilloid type 1. Hypertension 2006; 47: 609-614.

35. Touyz RM, He Y, Montezano AC, et al: Differential regulation of transient receptor potential melastatin 6 and 7 cation channels by ANG II in vascular smooth muscle cells from spontaneously hypertensive rats. Am J Physiol Regul Integr Comp Physiol 2006; 290: R73-R78.

36. Thilo F, Scholze A, Liu DY, Zidek W, Tepel M: Association of transient receptor potential canonical type 3 (TRPC3) channel transcripts with proinflammatory cytokines. Arch Biochem Biophys 2008; 471: 57-62.

37. Liu DY, Scholze A, Zhu ZM, et al: Transient receptor potential channels in essential hypertension. $J$ Hypertens 2006; 24: 1105-1114.

38. Liu DY, Thilo F, Scholze A, et al: Increased store-operated and 1-oleoyl-2-acetyl-sn-glycerol-induced calcium influx in monocytes is mediated by transient receptor potential canonical channels in human essential hypertension. $J$ Hypertens 2007; 25: 799-808.

39. Tong Q, Hirschler-Laszkiewicz I, Zhang W, et al: TRPC3 is the erythropoietin-regulated calcium channel in human erythroid cells. J Biol Chem 2008; 283: 10385-10395.

40. Stefanini M, Urbas JV, Urbas JE: Gaisböck's syndrome: its hematologic, biochemical and hormonal parameters. Angiology 1978; 29: 520-533.

41. Smith KJ, Bleyer AJ, Little WC, Sane DC: The cardiovascular effects of erythropoietin. Cardiovasc Res 2003; 59: 538-548.

42. Manjunatha H, Srinivasan K: Hypolipidemic and antioxidant effects of curcumin and capsaicin in high-fat-fed rats. Can J Physiol Pharmacol 2007; 85: 588-596.
43. Tani Y, Fujioka T, Sumioka M, Furuichi Y, Hamada H, Watanabe T: Effects of capsinoid on serum and liver lipids in hyperlipidemic rats. J Nutr Sci Vitaminol (Tokyo) 2004; 50: 351-355.

44. Bergdahl A, Gomez MF, Dreja K, et al: Cholesterol depletion impairs vascular reactivity to endothelin-1 by reducing store-operated $\mathrm{Ca}^{2+}$ entry dependent on TRPC1. Circ Res 2003; 93: 839-847.

45. Liu M, Huang W, Wu D, Priestley JV: TRPV1, but not $\mathrm{P} 2 \mathrm{X}$, requires cholesterol for its function and membrane expression in rat nociceptors. Eur J Neurosci 2006; 24: 1-6.

46. So I, Chae MR, Kim SJ, Lee SW: Lysophosphatidylcholine, a component of atherogenic lipoproteins, induces the change of calcium mobilization via TRPC ion channels in cultured human corporal smooth muscle cells. Int J Impot Res 2005; 17: 475-483.

47. Berthier A, Lemaire-Ewing S, Prunet C, et al: Involvement of a calcium-dependent dephosphorylation of BAD associated with the localization of Trpc-1 within lipid rafts in 7ketocholesterol-induced THP-1 cell apoptosis. Cell Death Differ 2004; 11: 897-905.

48. Beech DJ: Ion channel switching and activation in smoothmuscle cells of occlusive vascular diseases. Biochem Soc Trans 2007; 35: 890-894.

49. Kumar B, Dreja K, Shah SS, et al: Upregulated TRPC1 channel in vascular injury in vivo and its role in human neointimal hyperplasia. Circ Res 2006; 98: 557-563.

50. Takahashi Y, Watanabe H, Murakami M, et al: Involvement of transient receptor potential canonical 1 (TRPC1) in angiotensin II-induced vascular smooth muscle cell hypertrophy. Atherosclerosis 2007; 195: 287-296.

51. Kwan HY, Huang Y, Yao X: TRP channels in endothelial function and dysfunction. Biochim Biophys Acta 2007; 1772: 907-914.

52. Roberts LA, Connor M: TRPV1 antagonists as a potential treatment for hyperalgesia. Recent Patents CNS Drug Discov 2006; 1: 65-76.

53. Suri A, Szallasi A: The emerging role of TRPV1 in diabetes and obesity. Trends Pharmacol Sci 2008; 29: 29-36. 